\title{
Spinal cord stimulation: panacea for incurable diseases?
}

\author{
Jae Hang Shim \\ Department of Anesthesiology and Pain Medicine, School of Medicine, Hanyang University, Guri Hospital, Guri, Korea
}

A spinal cord stimulator is usually used as an electro-modulator for pain treatment. Shealy et al. [1] first reported the use of a spinal cord stimulator in an elderly male suffering with terminal bronchogenic carcinoma and right-sided chest pain in 1967. Since then, spinal cord stimulation (SCS) has been applied to many disease entities, such as neuropathic pain, failed back surgery syndrome, complex regional pain syndrome, and ischemic peripheral vascular disease [2-4]. The indications for SCS have been extended to include intractable angina pain, interstitial cystitis, intractable pain, headache, and non-painrelated applications such as refractory congestive heart failure, intractable spasticity, or treatment of cerebral vasospasm after subarachnoid hemorrhage [5-9]. SCS systems are composed of three components: leads/electrodes, a generator/power source, and a programmer/controller [5]. Leads can be divided into percutaneous leads and paddle leads. Small-sized rechargeable implanted batteries are becoming increasingly common. An electrical current is applied to the dorsal columns, creating a tingling sensation in the dermatomes whose afferent fibers traverse the regions being stimulated. The physician can control the degree, range of current, pulse width, and rate of the stimulation wave to optimize pain control.

In this issue of the Korean Journal of Anesthesiology, Ryu et al. [10] report the effect of cervical SCS for treatment of digit ulcers in a patient with Buerger's disease. Thromboangiitis obliterans is a type of segmental inflammatory vasculitis associated with severe pain and ulcers, which involves the small or medium-sized arteries and veins. Treatment of this painful ulcer is difficult and often involves amputation of the affected limb. This case report suggests that cervical SCS is a good choice for reducing pain and healing of an ulcer in patients with vasculitis. There are some reports of the effectiveness of SCS for patients with Raynaud's disease and Buerger's disease [11-13]. Cook et al. [14] reported using electronic stimulation of spinal nerves and the dorsal root in patients with a vascular disease of the limbs. Since then, SCS has been successfully used to treat atherosclerotic and vasospastic peripheral arterial disease. SCS improves microcirculation by inhibiting sympathetic vasoconstriction. SCS reduces pain in Buerger's disease by stimulating the secretion of a number of inhibitory neurotransmitters and promoting the secretion of gamma aminobutyric acid, substance $\mathrm{P}$, and serotonin at the spinal nerve dorsal horn $[15,16]$.

Wolter and Kieselbach [17] reported the long-term outcomes of patients with cervical SCS. Treatment indications included patients with causalgia, cervicobrachialgia, complex regional pain syndrome, phantom pain, and Raynaud's syndrome [18]. Pain reduction and paresthesia coverage were effective in almost all patients, and treatment satisfaction was high. No severe complications, such as neurological deficits or infection, were reported. They concluded that cervical SCS appears to be a safe and efficacious treatment option for upper limb neuropathic pain. Nevertheless, SCS has some disadvantages. The procedure for implanting SCS is invasive, so it is associated with complications such as infection, bleeding, and dural puncture [19]. The effectiveness of SCS is limited, and regular checks and continuous follow-ups are needed. Furthermore, SCS is expensive because of the limited medical insurance coverage in Korea. However, SCS is cost effective as compared with conservative management alone in cases with medical insurance coverage.

Advances in SCS technology and understanding of the

Corresponding author: Jae Hang Shim, M.D., Ph.D., Department of Anesthesiology and Pain Medicine, School of Medicine, Hanyang University, Guri Hospital, 249-1, Gyomun-dong, Guri 471-701, Korea. Tel: 82-31-560-2390, Fax: 82-31-563-1731, E-mail: jhshim@hanyang.ac.kr (c) This is an open-access article distributed under the terms of the Creative Commons Attribution Non-Commercial License (http:// creativecommons.org/licenses/by-nc/3.0/), which permits unrestricted non-commercial use, distribution, and reproduction in any medium, provided the original work is properly cited. 
mechanism will expand the field of SCS. As a result of the tremendous innovation in SCS, a number of new and exciting devices and therapies appear to be lifesavers for patients with intractable disease. The implanted narrow paddle leads, multichannel device with multi-polar leads, integrated accelerometer, sensing feedback technology, and alternate stimulation paradigms promise to increase efficacy and provide the opportunity to recover human life completely in the near future [9]. Consequently, developments in engineering and medical science will likely allow SCS to become a more useful tool for pain relief and expand the treatment spectrum.

\section{References}

1. Shealy CN, Mortimer JT, Reswick JB. Electrical inhibition of pain by stimulation in the dorsal columns: preliminary clinical report. Anesth Analg 1967; 46: 489-91.

2. Kumar K, Hunter G, Demeria D. Spinal cord stimulation in treatment of chronic benign pain: challenges in treatment planning and present status, a 22-year experience. Neurosurgery 2006; 58: 481-96.

3. De La Porte C, Van de Kelft E. Spinal cord stimulation in failed back surgery syndrome. Pain 1993; 52: 55-61.

4. Tesfaye S, Watt J, Benbow SJ, Pang KA, Miles J, MacFarlane IA. Electrical spinal-cord stimulation for painful diabetic peripheral neuropathy. Lancet 1996; 348: 1698-701.

5. Epstein LJ, Palmieri M. Managing chronic pain with spinal cord stimulation. Mt Sinai J Med 2012; 79: 123-32.

6. Mannheimer C, Augustinsson LE, Carlsson CA, Manhem K, Wilhelmsson C. Epidural spinal electrical stimulation in severe angina pectoris. Br Heart J 1988; 59: 56-61.

7. Gaul C, Jürgens T, May A. Concerning high cervical spinal cord stimulation for chronic cluster headache. Cephalalgia 2011;31: 1588-9.

8. Goellner E, Slavin KV. Cervical spinal cord stimulation may prevent cerebral vasospasm by modulating sympathetic activity of the superior cervical ganglion at lower cervical spinal level. Med Hypotheses 2009; 73: 410-3.

9. Levy RM. Spinal cord stimulation in 2020. Neuromodulation 2013; 16: 93-6.

10. Ryu SW, Jeon HJ, Cho SS, Choi RM, Yoon JS, Ko HS, et al. Treatment of digit ulcers in a patient with Buerger's disease by using cervical spinal cord stimulation. Korean J Anesthesiol 2013; 65: 167-71.

11. Issa MA, Kim CH. Cervical spinal cord stimulation with 5-column paddle lead in Raynaud's disease. Pain Physician 2012; 15: 303-9.

12. Wolter T, Kieselbach K. Spinal cord stimulation for Raynaud's syndrome: long-term alleviation of bilateral pain with a single cervical lead. Neuromodulation 2011; 14: 229-33.

13. Fabregat G, Villanueva VL, Asensio JM, De Andrés J, López D. Spinal cord stimulation for the treatment of Buerger disease: a report on 3 cases. Clin J Pain 2011; 27: 819-23.

14. Cook AW, Oygar A, Baggenstos P, Pacheco S, Kleriga E. Vascular disease of extremities. Electric stimulation of spinal cord and posterior roots. N Y State J Med 1976; 76: 366-8.

15. Linderoth B, Stiller CO, Gunasekera L, O'Connor WT, Franck J, Gazelius B, et al. Release of neurotransmitters in the CNS by spinal cord stimulation: survey of present state of knowledge and recent experimental studies. Stereotact Funct Neurosurg 1993; 61: 157-70.

16. Stanton-Hicks M, Salamon J. Stimulation of the central and peripheral nervous system for the control of pain. J Clin Neurophysiol 1997; 14: 46-62.

17. Wolter T, Kieselbach K. Cervical spinal cord stimulation: an analysis of 23 patients with long-term follow-up. Pain Physician 2012; 15: 20312.

18. Kim SJ, Kang G, Lee MG, Lee MK, Choi SS. Effective treatment of a digital ulcer and pain in a Raynaud's syndrome patient with using a spinal cord stimulator : a case report. Anesth Pain Med 2009; 4: 11-4.

19. Kunnumpurath S, Srinivasagopalan R, Vadivelu N. Spinal cord stimulation: principles of past, present and future practice: a review. J Clin Monit Comput 2009; 23: 333-9. 